\title{
The sacral exam—what is needed to best care for our patients?
}

\author{
Jean Gabriel Previnaire ${ }^{1} \cdot$ Marcalee Alexander $^{2}$
}

Received: 26 September 2019 / Accepted: 23 October 2019

(c) International Spinal Cord Society 2020

\begin{abstract}
Sacral reflexes are important to allow the SCI practitioner to gain information about the state of the sacral spinal cord segments. The presence of the bulbocavernosus and/or the anal wink reflex indicate an intact spinal reflex arc and reflex conal autonomic function (as part of the upper motor neuron syndrome); their absence defines a lower motor neuron syndrome. The assessment of sacral reflexes helps predict the type of bladder, bowel and sexual functions and the related therapeutic interventions. We suggest adding the sacral component of the International Standards for the Assessment of Autonomic Function after SCI (ISAFSCI) to the International Standards for the Neurologic Classification of Spinal Cord Injury (ISNCSCI) examination so there can be a detailed description of these important functions. As an alternative, the performance of sacral reflexes should be routinely required as part of the neurologic examination after SCI. Whether the sacral motor neuron system is classified as upper or lower motor neuron injury is also quite useful and as such should be present in the ISCNSCI.
\end{abstract}

\section{Introduction}

The International Standards for Neurological Classification of Spinal Cord Injury (ISNCSCI) were developed in 1982 by the American Spinal Injury Association (ASIA) to provide precision in the definition of neurological levels and extent of spinal cord injury (SCI) and achieve consistent and reliable data for patient care and research [1]. The ISNCSCI gives an accurate neurological level of injury (NLI) and is useful to prognosticate motor recovery [2]. The International Standards for the Assessment of Autonomic Function after SCI (ISAFSCI) were published in 2009 and designed to complement the ISNCSCI by documenting the impact of SCI on autonomic neural control of specific organ systems, including the sacral responses of bladder, bowel, and sexual function [3].

Sacral organ system functions depend on the level and severity of the SCI, and on the reflex activity of this sacral cord segment, i.e., the presence of an upper motor neuron

Jean Gabriel Previnaire

jean.previnaire@hopale.com

1 Spinal Unit, Centre Calve, Fondation Hopale, Berck-surMer, France

2 Department of PMR, University of Alabama at Birmingham School of Medicine, Birmingham, AL, USA
(UMN) lesion. In that regard, sacral reflexes in general and bulbocavernosus in particular are of great importance. In a recent survey, $55 \%$ of practitioners routinely determined the presence or absence of the bulbocavernosus reflex, and $48 \%$ routinely checked the anal wink reflex; a further $80 \%$ would be amenable to performing the bulbocavernosus reflex as part of the ISNCSCI exam [4].

\section{Methods}

Expert opinion and research of literature.

\section{Results}

Sacral reflexes are the contraction of the perineal muscles in response to stimulation of the perineum, urethra/bladder, or anus. Testing of the sacral reflexes assesses the integrity of the sacral spinal cord at S2 to S4 as well as the associated afferent and efferent pathways [5]. The afferent pathway of the reflex may be somatic, sympathetic, or parasympathetic, depending on the site of stimulation, but the efferent pathway is somatic and usually monitored in muscles innervated by the pudendal nerve $[6,7]$. Two of these reflexes are commonly used clinically: the bulbocavernosus reflex, elicited with compression of the glans penis or clitoris, by pulling pubic hairs or pulling on a catheter [4], and the anal 
wink which is the contraction of external anal sphincter, elicited with pinprick of the mucocutaneous junction of the anus or scratching a pin around the anus. In each case a response may be observed or palpated in the bulbocavernosus muscle or the anal sphincter (the contraction can also be felt with the examiner's finger in the anus). Reflex anal contraction from digital stretch (left and right) is also useful and easy to obtain.

Presence of sacral reflexes below the level of injury is key to determining a UMN lesion, especially when reflexes are triggered from sensory denervated skin, are brisk, or diffuse to other perineal muscles [8]. Absence of sacral reflexes defines a lower motor neuron (LMN) lesion.

UMN syndromes are usually found in cervical and higher thoracic NLI, and associate paralysis and spasticity, ankle clonus, and increased deep tendon reflexes, or Babinski sign [9]. LMN syndromes are found in lumbar and sacral NLI, typically in cauda equina syndromes, and are characterized by flaccid, atrophic, paralyzed lower limbs and absent lower limb deep tendon reflexes. Lower thoracic NLI affecting the conus medullaris is usually associated with a mixture of UMN and LMN pictures [2].

The type of lesion affecting the sacral area is important to know to help predict the type of sphincter functions [10]. For bladder and bowel, a UMN lesion is significantly correlated with detrusor overactivity and detrusor sphincter dyssynergia, rectal contraction and reflex stool leakage. As opposite, an LMN is associated with detrusor areflexia, flaccid paralysis of the striated sphincter and urinary stress incontinence, absent rectal contraction and passive leakage. For sexual life, a UMN lesion will promote reflex erection and projectile ejaculation in men and reflex lubrication in women, and a the presence of an LMN and the absence of sacral sensation will substantial decrease the likelihood of orgasm in both sexes compared with persons with all other injury types $[10,11]$. Therapeutic interventions will vary accordingly.

If one reflex is absent, we stress it is important to consider both the bulbocavernosus and the anal wink reflexes as one of them can be physiologically absent in a person [12]. Transient absence can also be observed in some physiologically cases, such as a full rectum inhibiting these reflexes or in case of spinal shock [13].

In the ISNCSCI examination, completeness of SCI is defined as the absence of sacral sparing (sensory or motor function in the most caudal sacral segments), whereas an incomplete injury is defined as the presence of sacral sparing [2]. However, despite the clinical importance of sacral autonomic function, neither the ISAFSCI nor the sacral reflexes provide sufficient information about the impact of injury on sacral autonomic function.

One weakness in the current ISNCSCI is the understanding of how to perform the anal exam. A recent study showed that there was a lack of knowledge about the definition of deep anal pressure with $20 \%$ reported they routinely applied firm pressure against the anal sphincter (as recommended in the 2011 revised ISNCSCI), 29\% placed firm pressure against the rectal wall, and $44 \%$ routinely applied pressure against the anal sphincter and the rectal wall [4]. This discrepancy is important because the innervation of the anal sphincter is somatic (S2-S4 through the pudendal nerve), while the innervation of the rectum is autonomic (S2-S4 via the parasympathetic nerve). This issue is crucial when we realize the anal exam is used to predict recovery of somatic neurologic status and as inclusion criteria in clinical trials.

There is also the issue of persons with incomplete sacral injuries that may be missed. If someone regains reflex function and/or sensation, there is a different possibility for recovery of bladder, bowel, and sexual function than without $[14,15]$.

As recommended by other investigators [4], we believe it is imperative to add more information to the ISNCSCI examination regarding sacral function. To accurately assess an individual's neurologic functioning after SCI, there are two possibilities.

(1) The preferred suggestion is to add the sacral component of the autonomic standards to the ISNCSCI examination so there can be a detailed description of these important functions or (2) to routinely require the performance of sacral reflexes as part of the examination so that this important prognosticating piece of data is not missed. We believe the bulbocavernosus is preferable; however, depending on the preference and the experience of the examiner if the anal wink is absent, the practitioner needs to then do the bulbocavernosus exam for confirmation. Whatever the option, anal exam with deep anal pressure must also be properly performed for the ISNCSCI classification.

\section{Conclusion}

In conclusion, sacral reflexes and determination of voluntarily anal sphincter contraction are important to allow the practitioner to gain information about the state of the sacral spinal cord segments. When present, it is indicative of intact spinal reflex arcs and reflex conal autonomic functions. In cervical and high thoracic NLI, UMN bladder, bowel, and sexual syndromes are generally found. Lower lumbo-sacral NLI or cauda equina will have a different presentation with areflexic LMN syndromes. Understanding and anticipation of these autonomic functions will help the patients' care. Systematic assessment will further help classify patient and facilitate patients' inclusion criteria for clinical studies.

For these reasons, there is no doubt that conal reflexes should be assessed and integrated into the ISNCSCI. We 
propose assessment of both reflexes if one is absent or a single if one is present, and the addition of information about remaining sacral function as per the ISAFSCI as part of the ISNCSCI examination. The type of sacral motor neuron system (UMN/LMN) is also quite useful and as such should be present in the ISCNSCI.

\section{Compliance with ethical standards}

Conflict of interest JGP has nothing to disclose. MA is author of the book "sexual sustainability".

Publisher's note Springer Nature remains neutral with regard to jurisdictional claims in published maps and institutional affiliations.

\section{References}

1. Association. ASI. Standards for neurological classification of spinal cord injury patients. Chicago: American Spinal Injury Association; 1982.

2. Kirshblum SC, Burns SP, Biering-Sorensen F, Donovan W, Graves DE, Jha A, et al. International standards for neurological classification of spinal cord injury (revised 2011). J Spinal Cord Med. 2011;34:535-46.

3. Alexander MS, Biering-Sorensen F, Bodner D, Brackett NL, Cardenas D, Charlifue $\mathrm{S}$, et al. International standards to document remaining autonomic function after spinal cord injury. Spinal Cord 2009;47:36-43.
4. Alexander M, Aslam H, Marino RJ. Pulse article: how do you do the international standards for neurological classification of SCI anorectal exam? Spinal Cord Ser Cases. 2017;3:17078.

5. Previnaire JG. The importance of the bulbocavernosus reflex. Spinal Cord Ser Cases. 2018;4:2.

6. Roberts MM. Neurophysiology in neurourology. Muscle Nerve 2008;38:815-36.

7. Uher EM, Swash M. Sacral reflexes: physiology and clinical application. Dis Colon Rectum. 1998;41:1165-77.

8. Maury. La paraplégie. Paris: Flammarion Médecine Sciences; 1981.

9. Doherty JG, Burns AS, O'Ferrall DM, Ditunno JF Jr. Prevalence of upper motor neuron vs lower motor neuron lesions in complete lower thoracic and lumbar spinal cord injuries. J Spinal Cord Med. 2002;25:289-92.

10. Previnaire JG, Soler JM, Alexander MS, Courtois F, Elliott S, McLain A. Prediction of sexual function following spinal cord injury: a case series. Spinal Cord Ser Cases. 2017;3:17096.

11. Alexander MS, Marson L. The neurologic control of arousal and orgasm with specific attention to spinal cord lesions: integrating preclinical and clinical sciences. Auton Neurosci. 2018; 209:90-9.

12. Blaivas JG, Zayed AA, Labib KB. The bulbocavernosus reflex in urology: a prospective study of 299 patients. J Urol. 1981;126:197-9.

13. Ko HY. Revisit spinal shock: pattern of reflex evolution during spinal shock. Korean J Neurotrauma. 2018;14:47-54.

14. Alexander MS, Carr C, Chen Y, McLain A. The use of the neurologic exam to predict awareness and control of lower urinary tract function post SCI. Spinal Cord 2017;55:840-3.

15. Sipski ML, Alexander CJ, Rosen R. Sexual arousal and orgasm in women: effects of spinal cord injury. Ann Neurol. 2001;49:35-44. 